\title{
Einführung der TARMED-Tarife oder die Doppelbödigkeit des Rationalen, das auf seiner Rückseite immer auch Irrsinn ist
}

\author{
H. H. Brunner
}

Was nicht produziert wurde, ist immer besser, als was produziert wird.

Marcel Duchamps

Die Einführung der TARMED-Tarife im KVG-Bereich scheint unmittelbar bevorzustehen; die aktuellen Verhandlungen drehen sich um die Frage des richtigen Starttaxpunktwertes und seiner kostenneutralen Steuerung in der Einführungsphase. Hier wird versucht, eine Standortbestimmung mit dem Ziel vorzunehmen, auf grundsätzliche, ungelöste Probleme dieser Taxpunktwertfestlegung hinzuweisen. Die Ausführungen beziehen sich weitgehend auf den KVG-Bereich; für die UV/MV/IV-Tarife ist der Taxpunktwert für die Ärzte geregelt, die dem entsprechenden Vertrag beitreten (Start-TPW Fr. 1.-).

\section{Ausgangslage}

Das KVG geht für die Festlegung der Tarife im KVG-Bereich vom Verhandlungsprimat aus: Die Vertragsparteien - Leistungserbringer auf der einen, Kostenträger auf der anderen Seite schliessen Verträge ab, die durch die Kantonsregierungen zu genehmigen sind. Der Entscheid kann nur Ja oder Nein sein. Im Rekursfall entscheidet der Bundesrat.

Dieses Dispositiv wurde bald einmal durch verschiedene Entwicklungen lädiert:

- Die mit dem KVG verfolgte Eindämmung des Kostenwachstums im Gesundheitswesen konnte durch eine Preispolitik nicht erreicht werden: Die Mengen liefen aus dem Ruder, um so dramatischer, je stärker die Preise gesenkt bzw. eingefroren wurden.

- Die Verordnungspraxis von EDI und Bundesrat war freundlich formuliert zurückhaltend; es gelang nicht, verbindliche Kosten/Preis/ Mengenkriterien zu verankern, ja nicht einmal eine korrekte Sammlung von Kostendaten, insbesondere aus den Spitälern, sicherzustellen. Den Gründen hierfür nachgehen $\mathrm{zu}$ wollen, würde bedeuten, sich in das Gebiet der Politastrologie zu begeben.

- Das EDI beschritt in der Folge immer mehr den Weg der Konkretisierung über die Rechtsprechung. Neben den grundsätzlichen staatsrechtlichen Fragen der richtigen Normstufe und des Legalitätsprinzips (EP-GPKSR-01, 327) sind auch ganz praktische Probleme mit diesem Vorgehen angesprochen: Rechtsprechung bezieht sich immer auf einen konkreten Fall; die Generalisierbarkeit der Erkenntnisse ist eine von juristischen Unwägbarkeiten belastete, dornenvolle Angelegenheit.

Seit etwa 1998 wurden diese Probleme aller offizieller Schönrednerei zum Trotz immer klarer erkennbar. Und damit begann der Aufstieg des Preisüberwachers zur dominanten Person der Eindämmung jeglichen Kostenwachstums: Die immer kritischere Situation ungenügender Vorgaben auf der Ebene Gesetz wie Verordnung und fehlender Daten bereitete den Weg für diesen 14. Nothelfer, der in der Schweiz den Heiligenschein des unabhängigen Preispolizisten uneingeschränkt sein eigen nennen darf. Kommt hinzu, dass diesem Aufstieg keine parteipolitischen Feindschaften zur damaligen Chefin des EDI im Wege standen.

\section{Preisüberwachung: Gesetz und Realität}

Tarifverträge gelten als Wettbewerbsabrede; deshalb müssen die Kantonsregierungen vor Genehmigung dieser Tarifverträge (wie auch vor Festsetzung eines hoheitlichen Tarifs im Falle des vertragslosen Zustandes!) die Preisüberwachung (PÜW) anhören (Art. 14 Abs. 1 PüG). Die Stellungnahme der PüW erfolgt in Form einer Empfehlung, die keinem Rekursverfahren unterliegt und damit nicht angefochten werden kann.

Der Bundesrat räumt der PüW den Status eines Sachverständigen (VwVG Artikel 12), seinen Empfehlungen den Charakter eines Sachverständigengutachtens ein (EP-GSK-SR-02, 339). Und dies ist nur ein Gebiet, wo PüW offensichtlich hohe Sachkompetenz hat.

Dies ist sehr bemerkenswert: Offensichtlich hat die Bundesverwaltung eine Effizienz, die weit grösser ist als diejenige aller anderen Länder, 


\section{Take-home-Messages}

- Die PüW ist zur massgeblichen Institution für die Festlegung der Taxpunktwerte im KVG-Bereich. Diese Entwicklung steht im Gegensatz zur Grundidee des KVG, das ein Vertragsprimat stipuliert und für Genehmigungsverfahren dem Ermessen der zuständigen kantonalen erheblichen Spielraum einräumt.

- Die Methode der PüW zur Ermittlung der (Start-)Taxpunktwerte basiert auf der Annahme eines «richtigen» Schweizerischen Taxpunktwertes um den herum die kantonalen Taxpunktwerte streuen. Diese Methode steht in konträrem Gegensatz zur Ermittlung der Starttaxpunktwerte nach dem Kostenneutralitätsprinzip, wie sie in den Verhandlungen zwischen santésuisse und den Kantonalen Ärztegesellschaften zur Anwendung kommt.

- Für die Genehmigungs- bzw. Rekursverfahren der Starttaxpunktwerte für die neuen TARMED-Tarife im KVG-Bereich sind hier aus rein systembezogener Betrachtung sachlich unlösbare Konflikte zu erwarten. Überdies wird in diesem Konflikt der letzte Rest betriebswirtschaftlicher Tarifberechnung eliminiert und zudem das vertraglich vereinbarte, vom Bund genehmigte Kostenneutralitätskonzept weitgehend ausgehölt.

die zu diesem Zweck - auch kleinere Länder Dutzende von Mitarbeitern und manchmal mehrere Sachverständigenräte bemühen müssen. Tu felix Helvetia gaude.

Die SGK Ständerat scheint diese Beurteilung nicht vollumfänglich teilen zu wollen, empfiehlt er doch in seinem Bericht vom 5. April 2002 dem Bundesrat, «den Berechnungsmodellen der Preisüberwachung auch andere Expertenmeinungen gegenüberzustellen und eine verstärkte Abwägung vorzunehmen (kein Automatismus)» (Empfehlung 5 EP-GPK-SR01). Der Bundesrat wiederum scheint von solchem nicht viel zu halten, fegt er doch die Empfehlung kurzangebunden mit der Einlassung vom Tisch, dass dies das Verfahren verlängern würde und das System der Tariffestsetzung auf den Kopf stellen würde, weil ja das Vertragsprimat gelten würde, das eben durch den Sachverständigen von der PüW über Bord gekippt wird. Da bleibt nur resigniert festzustellen, dass hier wirklich etliches auf dem Kopf steht, das sich vorher zudem noch in den eigenen Schwanz gebissen hat. So kann einem nur noch die Aussage des Bundesrates, «sich laufend über Evaluationen, Analysen und weitere Instrumente, welche für die Entscheidfindung in Tarifangelegenheiten von Nutzen sind» (EP-GPK-SR-02), zum stillen Trost gereichen.

Die Probleme werden dadurch noch verschärft, dass der Bundesrat nach Erfordernissen der EMRK für Beschwerdeentscheide gemäss KVG volle Kognition besitzt, also die Entscheide auch bezüglich ihres Ermessens überprüft. De facto werden damit kantonale Entscheide aus- gehebelt bzw. einer vollständigen materiellen Überprüfung offen gelegt. Damit soll verhindert werden, dass bloss formale Rückweisungen zu einem unendlichen Rezess von erneuten Entscheiden, erneuten Rekursen und erneuten Rückweisungen an die kantonale Vorinstanz führen. Juristisch vornehm heisst dies «Devolutiveffekt»; etwas pragmatischer meint dies: Wir sagen Euch, wie wir es haben vollen. Basta.

\section{Einführung des Physiotherapietarifs oder das immer noch nicht verinnerlichte Lehrstück preisüberwacherlichen Vorgehens}

Der am 1. Juli 1998 genehmigte Physiotherapietarif ist allen Teilen den TARMED-Tarifen analog: Einheitliche schweizerische Tarifstruktur, Taxpunktwerte in den Kantonen auszuhandeln, wahrscheinlich erfolglose Verhandlungen in einer gewissen Zahl von Kantonen, Festlegung durch die Kantonalen Regierungen (KVG Art. 47 Abs. 1), Anfechtung der Entscheide meist gleichzeitig durch beide Vertragsparteien mit gegenläufigen Zielsetzungen, Entscheid des Bundesrates.

Der Bundesrat bzw. die Preisüberwachung gingen für die Entscheide von einem nationalen Modelltaxpunktwert von Fr. -.94 aus (TPW im UV/MV/IV-Bereich Fr. 1.-!). Die Begründung, weshalb der von der MTK errechnete, betriebswirtschaftlich korrekte Taxpunktwert von Fr. 1.abgesenkt wurde, erschliesst sich dem minimal rationale Argumentationen verlangenden Leser nicht. Basierend auf einer Formel, die vor allem Lohn und Mietstrukturen berücksichtigt, zweifelsohne die medizinökonomischen Kenngrössen par excellence, wurden dann die kantonalen Taxpunktwerte als Streuung um diesen «nationalen» Taxpunktwert ermittelt. Es muss erstaunen, dass nach dieser Methode in einem Raum wie der Nordostschweiz mit wirtschaftlich relativ homogenen Bedingungen die ermittelten Taxpunktwerte von Fr. - .90 bis 1.03 streuen.

Entscheidend ist nun, dass die Verhandlungen zwischen Kantonalen Gesellschaften und den lokal Zuständigen von santésuisse auf einem völlig anderen Modell beruhen: Sie orientieren sich am Primat der Kostenneutralität für die Einführungsphase, basierend auf einem Vergleich der Leistungen bzw. von Leistungspaketen der aktuellen Kantonalen Tarife und des neuen kantonalen TARMED-Tarifs. Dieses Vorgehen muss zwangsläufig zu anderen Resultaten führen, die aber solange hinfällig sind, als der Bundesrat sich ausschliesslich auf die Rechnungsmodelle der PüW abstützt. Die 


$\begin{array}{ll}\begin{array}{l}\text { Abkürzungen } \\ \text { EP-GPK-SR-01 }\end{array} & \begin{array}{l}\text { Aufsichtseingabe der Kantone zur Entscheidungspraxis des Bundesrates } \\ \text { bei Beschwerden gegen Tarifentscheide der Kantonsregierungen in der } \\ \text { Krankenversicherung vom 5. April 2002 }\end{array} \\ \text { EP-GPK-SR-02 } & \begin{array}{l}\text { Stellungnahme des Bundesrates vom 30. September 2002 } \\ \text { PüG }\end{array} \\ \text { EMRK } & \begin{array}{l}\text { Preisüberwachungsgesetz vom 20. Dezember 1985 (SR 171.11) } \\ \text { Europäische Menschenrechtskonvention vom 4. November 1950 } \\ \text { (SR 0.101) }\end{array} \\ \text { KVG } & \begin{array}{l}\text { Bundesgesetz über die Krankenversicherung vom 18. März 1994 } \\ \text { (SR 832.10) }\end{array} \\ & \end{array}$

PüW selbst ist, wie immer wieder von ihr betont, fest entschlossen, ihre Sicht durchzusetzen, dies auf der kantonalen Ebene wie auch im Rekursfall auf eidgenössischer Ebene.

Der Bundesrat hat kein Abgehen von seiner Position signalisiert. Dies bedeutet, dass die gesamten Rekursentscheide, die noch vor der künftigen Totalrevision der Bundesrechtspflege über die Bühne gehen werden, nach diesem Muster ablaufen werden. Der Bundesrat bleibt damit in der Position der einzigen Rekursinstanz, der zudem noch der Gesetzesvollzug, die Verordnungsgebung obliegt. Anlass, einen der vielen Mythen über dieses Land kritisch zu reflektieren, vorliegend der, die Schweiz sei ein Ausbund demokratischer Gewaltentrennung.
Die Reform der Bundesrechtspflege sieht u.a. vor, dass der Bundesrat von seiner inzwischen atypischen Funktion der Rechtsprechung im KVG entlastet und diese Aufgabe an das künftige Bundesverwaltungsgericht delegiert werden wird. Von dieser sinnvollen Reform wird die Einführung der TARMED-Tarife nicht profitieren.

\section{Konklusion - oder was nun?}

Verschiedene Kontakte haben gezeigt, dass sich nur wenige der Verantwortungsträger dieser Problematik bewusst sind, vielleicht auch bewusst sein wollen. Die FMH wird den Vorsteher des EDI, Bundespräsidenten Pascal Couchepin, bitten, eine Sitzung der Beteiligten einzuladen mit dem Ziel, die übelsten der angedeuteten Entwicklungen zu verhindern. Gelingt dies nicht, könnte TARMED im $K V G$-Bereich wirklich zu dem immer wieder heraufbeschworenen Absturz führen. Nicht wegen Reengineerings II und seiner nicht in allen Teilen erfüllbaren Hoffnungen, nicht wegen der Dignitätserhebung, sondern wegen gesetzlicher und administrativer Randbedingungen, die einen betriebswirtschaftlich berechneten Tarif, der schon ordentlich Schaden gelitten hat, definitiv zu einem der Hölle entstiegenen Monster zu pervertieren drohen. 